\title{
CAPÍTULO 62
}

\section{MODIFICAÇÃO DOS HÁBITOS ALIMENTARES E DA SAÚDE MENTAL EM ADULTOS NA PANDEMIA DA COVID-19}

\author{
DOI 10.4322/978-65-995353-2-1.c62
}

\author{
$\underline{\text { Lucas Araújo Ferreira }}^{\mathbf{1}}$, Amanda Vitória do Nascimento da Silva ${ }^{2}$, Ana Jhennyfer da Silva \\ Moreira $^{3}$, Ariel Christine dos Anjos Solano ${ }^{4}$, Bruna Raciele de Sousa Nascimento ${ }^{5}$, Carliane \\ Cardoso dos Reis ${ }^{6}$, Juliana Oliveira Ferreira ${ }^{7}$, Laisy da Cruz Corrêa ${ }^{8}$, Laisy Nazaré Araújo \\ da Cunha9, Yasmin Carvalho Barata Pereira ${ }^{10}$ \\ ${ }^{1}$ Universidade Federal do Pará (UFPA), (lucas.parasitologist@gmail.com); \\ 2Universidade Federal do Pará (UFPA), (amandavitoria.nascm@gmail.com); \\ 3Universidade Federal do Pará (UFPA), (jhenny20ana@gmail.com); \\ ${ }^{4}$ Universidade Federal do Pará (UFPA),(ari.solano97@ gmail.com); \\ ${ }^{5}$ Universidade Federal do Pará (UFPA), (b.raciele.sousa@gmail.com); \\ Universidade Federal do Pará (UFPA), (reiscarli1@gmail.com); \\ ${ }^{7}$ Universidade Federal do Pará (UFPA), (julianaoliveiraferreira27@ gmail.com); \\ ${ }^{8}$ Universidade Federal do Pará (UFPA), (laisy_correa@hotmail.com); \\ 9 Universidade Federal do Pará (UFPA), (laisy.cunha33@gmail.com); \\ ${ }^{10}$ Universidade Estácio de Sá, (yasmincbarata@gmail.com)
}

\begin{abstract}
Resumo
Objetivo: Analisar, através de revisão literária, as alterações comportamentais e modificações nos hábitos alimentares desencadeadas pelo isolamento social durante a pandemia do covid-19. Método: Realizada pesquisa bibliográfica na base de dados SciELO (Scientific Electronic Library Online) e Google Scholar com as palavras-chaves hábitos alimentares, comportamento alimentar, pandemia, COVID-19 e seus correlatos em inglês. Mediante a pesquisa, foram selecionados 4 artigos, com o período de publicação delimitado de 2020 a 2021 e nos idiomas português e inglês. Resultados: Os estudos apresentados evidenciaram o agravamento do estado da saúde mental de pessoas que já apresentavam algum transtorno psicossomático. Além disso, a ingestão alimentar de produtos industrializados aumentaram durante o isolamento social, alterando gravemente os hábitos alimentares, que, relacionado às alterações da saúde mental durante o isolamento social, pode contribuir para o desencadeamento de modificações perigosas no estado nutricional e mental do indivíduo. Conclusão: Destarte, vê-se a necessidade de novos estudos que avaliem e relacionem o comportamento alimentar e mental durante a pandemia de Covid-19.
\end{abstract}

Palavras-chave: Hábitos alimentares; Comportamento Alimentar; Pandemia; Covid-19

Área Temática: Ciências da Saúde

E-mail do autor principal: lucas.parasitologist@ gmail.com

\section{INTRODUÇÃO}

Neste novo cenário epidemiológico, em dezembro de 2019, um novo vírus, o SARS-CoV-2 (Severe Acute Respiratory Syndrome Coronavirus 2) classificado na família de coronavírus, Pesquisa e Extensão 
responsável por causar a COVID-19 foi identificado no leste asiático, mais precisamente na cidade de Wuhan, na China (SANTOS; AYRES, 2021). Por sua grande facilidade de contágio, causou grande preocupação para a saúde da população mundial, e, devido isso, em março de 2020 a Organização Mundial da Saúde declarou o surto do novo coronavírus como "Emergência de Saúde Pública de Importância Internacional”. As recomendações de segurança das autoridades de saúde foram baseadas em medidas restritivas de circulação de pessoas, como a implementação de distanciamento social com objetivo de frear a disseminação do vírus (MAYNARD et al., 2021).

Desde o início, a pandemia do COVID-19 mudou consideravelmente as vidas de cada indivíduo, alterando e afetando o cotidiano, o trabalho e o bem estar social ocasionando disfunções e impactos na saúde mental (ALONZI; TORRE; SILVERSTEIN, 2020). Diversos grupos sentem o impacto que a pandemia causou, principalmente na saúde mental, e é difícil delimitar e montar intervenções para um grupo específico (ALONZI; TORRE; SILVERSTEIN, 2020). Em vista disso, indivíduos com dificuldades na saúde mental são grupos susceptíveis, pois apresentam mudanças no lazer e nos cuidados da saúde mental (ALONZI; TORRE; SILVERSTEIN, 2020).

De acordo com o boletim epidemiológico fornecido pelo Ministério da Saúde, até o mês de fevereiro de 2021 foram confirmados mais de 113 milhões de casos de COVID-19 no mundo, sendo os Estados Unidos o país com maior número de casos, mais de 28 milhões, seguido pelo Brasil com mais de 10 milhões de casos confirmados (BRASIL, 2021).

A pandemia da COVID-19 causou e ainda causa incertezas e inseguranças na população, e por conseguinte pode desencadear sinais e sintomas de problemas psicológicos e, consequentemente doenças mentais no qual estão inseridas a depressão, ansiedade e estresse traumático (BANNA, et al., 2020).

Os transtornos mentais albergam certo sofrimento psiquiátrico ocasionando problemas de sono e vigília, e tudo isso altera o cotidiano, a saúde mental e qualidade de vida dos indivíduos (BARROS, et al., 2020). Ainda segundo Barros et al. (2020) tais transtornos mentais podem agravar ou ser um fator de risco para o surgimento de doenças crônicas não transmissíveis e, por conseguinte doenças de cunho viral, no qual no caso de epidemias e o isolamento total, podem ampliar o problema e aumentar os quadros de doenças mentais na população (BARROS, et al., 2020).

Os pacientes que se encontram com suspeita ou já estão infectados pelo coronavírus, podem sentir medo do que possa vir a acontecer, e aos que estão em quarentena, os sentimentos de tédio, solidão e raiva podem surgir e afetar o fator emocional (DUARTE, et al., 2020).

O surgimento de incertezas sobre como conter o número de casos, e sobre o impacto que a doença poderia causar, afetou a saúde mental de cada pessoa mundialmente (SCHIMIDT, et al., 2020). Além disso, as notícias falsas, também chamadas de fake news, sobre o processo infeccioso, Pesquisa e Extensão 
medidas de prevenção ou até as orientações sobre os cuidados sanitários, circulam rapidamente e esse equívoco afeta ainda mais o estado psicológico (SCHIMIDT, et al., 2020).

A pandemia apresentou fatores positivos que podem ser levados em consideração, como ter mais contato com a família, além de ter proporcionado a oportunidade de trabalhar remotamente, ou seja, utilizando meios digitais como usar o computador em vídeo chamadas ou conferências online, mas os fatores psicológicos negativos predominam (LEUCHT, et al., 2021). E como relata Barros et al. (2020) pessoas diagnosticadas com transtornos mentais têm mais chance de ter agravos elevados de estresse e sofrimento psíquico no decorrer da pandemia da COVID-19, ao contrário do que um indivíduo sem transtornos diagnosticados (BARROS, et al., 2020).

A taxa de infecção do SARS-CoV-2 resultou num aumento expressivo, visto pelo número de casos confirmados, e nesse novo cenário pandêmico cuidados e modificações no estilo de vida foram necessários para manter a integridade na saúde da população (DURÃES, et al., 2020). Logo, algumas medidas foram estabelecidas pelo poder governamental, sob a cautela na diminuição da curva de crescimento do número de casos, tais como distanciamento social com restrição de circulação de pessoas em escolas, universidades, teatros, bares, restaurantes e empresas ou serviços de caráter não essencial (DURÃES, et al., 2020).

E com isso houveram mudanças psicológicas como transtornos de ansiedade, sentimentos de medo e tristeza e alteração de humor; pesquisas afirmam que essas modificações causadas pelo vírus podem estar relacionadas com o sistema imunológico e com neurotransmissores, no qual ocasiona mudanças também no comportamento alimentar da população, tanto no aumento da sensação de fome como também no aumento de apetite, causando quadros de excesso de peso e obesidade (OLIVEIRA, et al., 2021; SILVA, et al., 2020).

Além disso, no que pode-se referir ao consumo alimentar com relação ao mapa da fome, o Brasil obteve grande avanço, pois algumas políticas públicas foram criadas justamente para que indivíduos em condições de vulnerabilidade socioeconômica não perecessem (SILVA, et al., 2020). Logo, dentre os programas criados estão o Bolsa Família, e com esse programa houve a redução de $25 \%$ da pobreza extrema, ou seja, muitas famílias conseguiram sair de uma condição exacerbada de fome (SILVA, et al., 2020).

Apesar do Brasil ter tido avanço positivo no mapa da fome a décadas atrás, isso mudou durante a pandemia da COVID-19, em que famílias de baixa renda estavam em condições de insegurança e vulnerabilidade alimentar, e por conta disso houveram modificações nos hábitos alimentares (SILVA, et al., 2020). De acordo com Durães et al. (2020) o consumo de alimentos e sua comercialização foram afetados, gerando mudanças no aspecto econômico e social, no qual muitas famílias tiveram que optar pelo consumo de alimentos ultraprocessados devido seu baixo custo, maior durabilidade e Pesquisa e Extensão 
dentro do orçamento familiar, e o baixo consumo de alimentos in natura como frutas, legumes e verduras foi percebido (DURÃES, et al., 2020).

A obesidade é considerada uma questão multifatorial, e durante a pandemia foi observado crescimento no número de casos de obesidade, e esse fato se intensificou no decorrer da pandemia. Durante a Pesquisa de Vigilância de Fatores de Risco e Proteção para Doenças Crônicas por Inquérito Telefônico de 2019 (VIGITEL), foi observado certo aumento, muito significativo da obesidade entre os brasileiros nos últimos anos, partindo de 11,4\% em 2006 para 20,3\% em 2019 (BRASIL, 2019).

Em detrimento disso, algumas mudanças nas escolhas alimentares durante a pandemia estão relacionadas com esse crescimento ainda mais acelerado da obesidade, no qual está relacionado com os aspectos emocionais, refletindo em um comer transtornado, ou seja, escolhas alimentares não saudáveis, comer sem controle, substituições de refeições por lanches rápidos, serviços de entrega e alto consumo de alimentos ultraprocessados (SMAIRA, et al., 2021).

Desde o começo das restrições causadas pela pandemia da COVID-19, aumentou-se o consumo de alimentos caracterizados como junk food, e isso resultou num hábito alimentar desregulado com pouco consumo de alimentos in natura, em que o público afetado é variado, desde crianças, adolescentes até aos adultos, que tiveram seu estado nutricional alterado comprometendo sua condição de saúde (POELMAN, et al., 2021).

\section{MÉTODO}

O presente estudo trata-se de uma revisão sistemática da literatura, realizada durante o mês de agosto de 2021. Para sua construção, foram selecionados 04 artigos científicos, consultados nas bases de dados SciELO (Scientific Electronic Library Online) e PubMed, e para a busca, utilizou-se os respectivos descritores "hábitos alimentares (Eating Habits), comportamento alimentar (Feeding behavior), pandemia, (Pandemic) e COVID-19". Foram considerados como critérios de inclusão: Artigos científicos publicados em periódicos durante o período de 2020 a agosto de 2021, nos idiomas português e inglês, pertinentes e correspondentes ao objetivo do trabalho. Quanto aos critérios de exclusão, foram descartados todos os artigos que não obedeciam aos critérios acima citados, além de livros e diretrizes. Os artigos selecionados foram lidos integralmente e organizados em planilhas para melhor análise e construção de ideias de caráter científico, crítico e reflexivo baseadas em diferentes fontes textuais produzidas por diferentes autores que permitem subsidiar as questões aqui argumentadas. Em relação aos aspectos éticos, o presente estudo poderia apresentar como risco, a não citação dos autores dos artigos selecionados, não apresentando a correta referência dos mesmos. Para evitar a ocorrência deste risco, foi adotado o uso de planilhas que catalogaram Pesquisa e Extensão 
todos os estudos selecionados, com todas as informações de autores, ano, título e link de acesso direto à revista ao qual o artigo está disponível. Como benefício desta pesquisa, observa-se a grande contribuição que a mesma oferece à comunidade científica, visto que é um tema atual, relevante e ainda pouco estudado devido a conjuntura pandêmica atual.

\section{RESULTADOS E DISCUSSÃO}

Tabela 1- Resultados bibliográficos encontrados na literatura.

\begin{tabular}{|c|c|c|c|c|}
\hline $\begin{array}{c}\text { Título do } \\
\text { artigo }\end{array}$ & $\begin{array}{l}\text { Tipo de } \\
\text { estudo }\end{array}$ & Metodologia & Resultados & Conclusão \\
\hline $\begin{array}{l}\text { Impacto da } \\
\text { pandemia de } \\
\text { coronavírus } \\
\text { (COVID-19) } \\
\text { no } \\
\text { comportament } \\
\text { o alimentar. } \\
(2021)\end{array}$ & $\begin{array}{l}\text { Estudo } \\
\text { transversal } \\
\text { (Nível de } \\
\text { evidência 1B) }\end{array}$ & $\begin{array}{l}\text { Os dados derivam de um projeto } \\
\text { aprovado pelo Comitê de Ética da } \\
\text { Universidade Federal do Estado do } \\
\text { Rio de Janeiro. Para a coleta foram } \\
\text { incluídos adultos com idade igual ou } \\
\text { superior a } 20 \text { anos e menor que } 59 \\
\text { anos, residentes no Brasil de ambos os } \\
\text { sexos. Estes foram participaram por } \\
\text { meio de um formulário eletrônico } \\
\text { disponibilizado em meios de } \\
\text { comunicação e redes midiaticas com o } \\
\text { Instagram, Facebook e Whatsapp. O } \\
\text { questionário era constituído em duas } \\
\text { partes, a qual uma se referia acerca do } \\
\text { consumo e hábitos alimentares e a } \\
\text { outra era voltada sobre a COVID-19. } \\
\text { Com o preenchimento dos dados foi } \\
\text { realizada uma análise descritiva à } \\
\text { obtenção dos resultados das variáveis } \\
\text { qualitativas e de mediana às } \\
\text { quantitativas. }\end{array}$ & $\begin{array}{l}\text { Das } 186 \text { pessoas que tiveram } \\
\text { sua participação validada, } \\
\text { observou-se a prevalência do } \\
\text { público feminino, da faixa } \\
\text { etária de } 20 \text { a } 29 \text { anos, dos } \\
\text { residentes do estado do Rio } \\
\text { de Janeiro, com o isolamento } \\
\text { social parcial e não } \\
\text { considerado do grupo de } \\
\text { risco. Ainda, apontou-se que } \\
\text { houve uma piora acerca dos } \\
\text { hábitos alimentares durante a } \\
\text { pandemia, assim como na } \\
\text { saúde mental, além de um } \\
\text { ganho de peso. Ao comparar } \\
\text { o antes e o depois da } \\
\text { pandemia, os hábitos } \\
\text { alimentares sofreram um } \\
\text { impacto significativo, } \\
\text { fazendo com que ocorresse } \\
\text { uma mudança no padrão e no } \\
\text { comportamento alimentar } \\
\text { sob a possível influência da } \\
\text { restrição social e da saúde } \\
\text { mental. }\end{array}$ & $\begin{array}{l}\text { Como conclusão, o estudo } \\
\text { indica que o confinamento } \\
\text { domiciliar tem um efeito } \\
\text { adverso no comportamento } \\
\text { alimentar, e que mesmo } \\
\text { apresentando uma amostra } \\
\text { de baixa representatividade } \\
\text { infere que os achados } \\
\text { possam auxiliar no } \\
\text { desenvolvimento } \\
\text { recomendações de } \\
\text { nutricionais com o intuito } \\
\text { de manter a saúde em época } \\
\text { de isolamento social e } \\
\text { compreender as } \\
\text { perspectivas associados à } \\
\text { alimentação e os fatores } \\
\text { que a interferem. }\end{array}$ \\
\hline $\begin{array}{l}\text { Food } \\
\text { consumption } \\
\text { and anxiety } \\
\text { among the } \\
\text { adult } \\
\text { population } \\
\text { during the } \\
\text { COVID-19 } \\
\text { pandemic in } \\
\text { Brazil (2020) }\end{array}$ & $\begin{array}{l}\text { Estudo } \\
\text { descritivo } \\
\text { (nível de } \\
\text { evidência 6) e } \\
\text { Estudo } \\
\text { transversal } \\
\text { (nível de } \\
\text { evidência 1B). }\end{array}$ & $\begin{array}{l}\text { No estudo foram incluídos adultos } \\
\text { brasileiros de } 18 \text { a } 59 \text { anos de ambos } \\
\text { os sexos com sintomas ou não da } \\
\text { COVID-19. Para a coleta de dados } \\
\text { utilizou-se um formulário online } \\
\text { divulgado em mídias sociais. O } \\
\text { questionário constou com elementos } \\
\text { de investigação acerca dos aspectos } \\
\text { sociodemográficos e antropométricos; } \\
\text { sintomas, histórico de contato com o } \\
\text { vírus, modificações de hábitos } \\
\text { alimentares e atividade física no } \\
\text { período de isolamento social, } \\
\text { consumo de alimentos durante o } \\
\text { confinamento e sintomas de } \\
\text { ansiedade. }\end{array}$ & $\begin{array}{l}1000 \text { questionários foram } \\
\text { sancionado, obtendo uma } \\
\text { prevalência de participantes } \\
\text { da região nordeste, entre a } \\
\text { faixa etária média de } 31 \text { anos } \\
\text { e do sexo feminino. No artigo } \\
\text { é evidenciado que } 84 \% \text { da } \\
\text { amostra demonstrou } \\
\text { ansiedade, sendo } \\
\text { majoritariamente a ansiedade } \\
\text { grave. A partir da análise do } \\
\text { Questionário de Frequência } \\
\text { Alimentar (QFA), notou-se } \\
\text { que pessoas sem ansiedade } \\
\text { consumiram com maior } \\
\text { regularidade os alimentos } \\
\text { pertencentes ao grupo } \\
\text { saudáveis em comparação }\end{array}$ & $\begin{array}{l}\text { Em suma, o artigo mostra } \\
\text { os danos causados pela } \\
\text { pandemia na saúde, em } \\
\text { especial a mental. Concluiu } \\
\text { também que houve } \\
\text { alterações no tocante à } \\
\text { dieta, tendo um aumento } \\
\text { das preparações das } \\
\text { refeições em casa em razão } \\
\text { do isolamento social e o } \\
\text { aumento de alimentos } \\
\text { saudáveis nos voluntários } \\
\text { que não tiveram ansiedade. }\end{array}$ \\
\hline
\end{tabular}




\begin{tabular}{|c|c|c|c|c|}
\hline & & & $\begin{array}{l}\text { àqueles que tiveram algum } \\
\text { nível de ansiedade. } \\
\text { Ainda, destacou que no grupo } \\
\text { dos alimentos não saudáveis } \\
\text { houve uma predominância da } \\
\text { ingestão de doces, embutidos } \\
\text { e refrigerantes, a qual foram } \\
\text { associados estatisticamente a } \\
\text { indivíduos com ansiedade, } \\
\text { observando a interferência do } \\
\text { transtorno psicológico no } \\
\text { comportamento alimentar. }\end{array}$ & \\
\hline $\begin{array}{l}\text { The COVID- } \\
19 \text { Pandemic } \\
\text { and changes in } \\
\text { adult Brazilian } \\
\text { lifestyles: a } \\
\text { cross-sectional } \\
\text { study, 2020. } \\
(2020)\end{array}$ & $\begin{array}{l}\text { Estudo } \\
\text { transversal } \\
\text { (Nível de } \\
\text { evidência 1B) }\end{array}$ & $\begin{array}{l}\text { Por intermédio de um inquérito de } \\
\text { saúde virtual "ConVid, Pesquisa de } \\
\text { Comportamento"'a coleta de dados } \\
\text { utilizou pessoas na idade de } 18 \text { anos } \\
\text { ou mais, residentes no território } \\
\text { brasileiro durante a pandemia de } \\
\text { COVID-19. Nesse questionário os } \\
\text { achados referem-se a respeito das } \\
\text { questões sociodemográficas, o } \\
\text { consumo de cigarro e bebidas } \\
\text { alcoólicas, hábitos alimentares, } \\
\text { atividade física, sedentarismo e uso de } \\
\text { meios televisivos e de computadores. }\end{array}$ & $\begin{array}{l}\text { Na amostra final de } 45.16, \\
\text { percebe-se o aumento da } \\
\text { ingestão de bebidas } \\
\text { alcoólicas em decorrência da } \\
\text { ansiedade e tristeza, do medo, } \\
\text { insegurança, bem como do } \\
\text { risco de morte e ainda há a } \\
\text { redução do consumo dos } \\
\text { alimentos saudáveis durante } \\
\text { a restrição social acometida } \\
\text { pela pandemia da COVID- } \\
\text { 19. Foi atentado que o } \\
\text { consumo de alimentos não } \\
\text { saudáveis durante a semana } \\
\text { com dois dias ou mais, } \\
\text { aumentou distou } \\
\text { significativamente. Atrelado } \\
\text { a isso, destacou-se a } \\
\text { diminuição considerável de } \\
\text { atividades físicas e um } \\
\text { sedentarismo expressivo } \\
\text { associado ao tempo de uso de } \\
\text { televisão, computador e } \\
\text { tablet. }\end{array}$ & 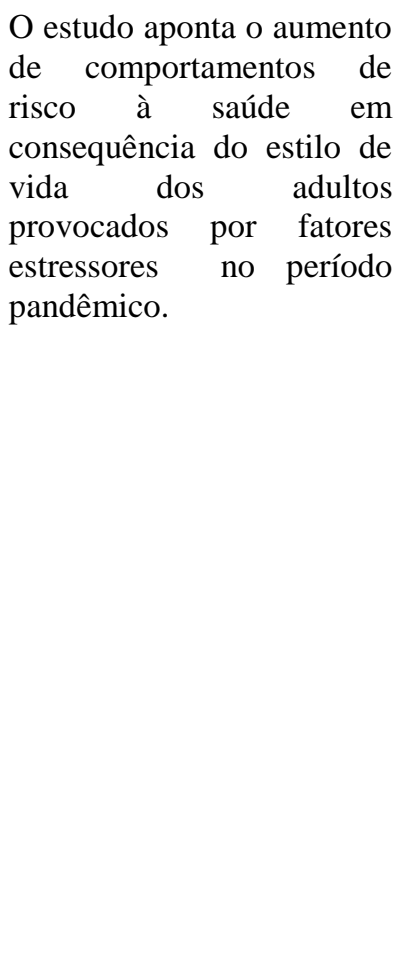 \\
\hline $\begin{array}{l}\text { Eating } \\
\text { behavior in } \\
\text { times } \\
\text { Covid-19 by } \\
(2021)\end{array}$ & $\begin{array}{l}\text { Estudo } \\
\text { longitudinal } \\
\text { prospectivo } \\
\text { (Nível de } \\
\text { evidência 2B) }\end{array}$ & $\begin{array}{l}\text { A pesquisa teve início após a } \\
\text { aprovação do Comitê de Ética e } \\
\text { Pesquisa, sendo efetuada em dois } \\
\text { momentos. No estudo foram incluídos } \\
\text { indivíduos maiores de } 18 \text { anos do } \\
\text { feminino e do masculino que } \\
\text { consentiram responder } \\
\text { questionários. }\end{array}$ & $\begin{array}{l}\text { Depois do levantamento e da } \\
\text { análise dos dados obtidos, } \\
\text { notou-se um aumento na } \\
\text { ingestão de alimentos } \\
\text { ultraprocessados mesmo que } \\
\text { o aumento do consumo de } \\
\text { verduras,frutas e legumes } \\
\text { tenha sido significativo, visto } \\
\text { que as emoções podem } \\
\text { interferir no comportamento } \\
\text { alimentar e, por } \\
\text { consequência, dar } \\
\text { preferência a alimentos que } \\
\text { contenham bastante } \\
\text { carboidrato as quais causam } \\
\text { sensações de relaxamento e } \\
\text { conforto. }\end{array}$ & $\begin{array}{l}\text { Teve-se comportamentos } \\
\text { compensatórios positivos e } \\
\text { negativos entre a população } \\
\text { estudada. No positivo } \\
\text { destacam-se o crescimento } \\
\text { da ingestão de alimentos } \\
\text { preparados em casa e o } \\
\text { aumento do exercício } \\
\text { físico, já no negativo } \\
\text { apontou-se uma crescente } \\
\text { no consumo de bebidas } \\
\text { alcoólicas e um comer } \\
\text { atrelado a emoções. }\end{array}$ \\
\hline
\end{tabular}

Fonte: Autores (2021).

Dos artigos selecionados, todos foram do tipo transversal, dois foram uma pesquisa por meio de redes sociais e um por intermédio de um inquérito de saúde virtual. Diante dos resultados, de 
acordo com a pesquisa de Santos; Ayres, (2021) realizada com 186 pessoas, sendo a maioria composta pelo público feminino, na faixa etária de 20 a 29 anos de idade. Verificou-se que houve uma piora acerca dos hábitos alimentares durante a pandemia, pois o consumo de refeições prontas industrializadas $(17,74 \%)$, biscoitos e lanches prontos $(38,17 \%)$ aumentou em comparação ao consumo durante a pandemia (refeições prontas industrializadas, biscoitos e lanches prontos, respectivamente $25,81 \%$ e 45,16\%) (SANTOS; AYRES, 2021).

As incertezas da COVID-19 ameaçam não só a saúde física das pessoas, como também a saúde mental, estudos apontam que respostas psicológicas e emocionais desencadeadas pela pandemia podem aumentar o risco de desenvolver uma alimentação disfuncional (LI et al., 2020; MONTEMURRO 2020; WANG et al., 2020 Apud OLIVEIRA et al., 2021).Os autores Santos; Ayres, (2021) observaram ainda, agravos na saúde mental, além de ganho de peso. Ademais, segundo o estudo de Maynard et al., (2021) o qual analisou o padrão alimentar e a ansiedade da população adulta durante o surto do Coronavírus no Brasil, o público apresentou faixa etária média de 31ะ15,37, sendo a maior parte do sexo feminino, foi evidenciado que $84 \%$ da amostra demonstrou ansiedade, sendo caracterizada como ansiedade grave, além disso, destacou-se que, no grupo dos alimentos não saudáveis houve uma predominância da ingestão de doces, embutidos e refrigerantes, os quais foram associados estatisticamente a indivíduos com ansiedade, observando a interferência do transtorno psicológico no comportamento alimentar. De acordo com esses artigos é possível notar que no momento da quarentena, ocorreram graves mudanças tanto na saúde mental quanto em relação aos hábitos alimentares, fatores que influenciam também no aumento do ganho de peso (MAYNARD et al., 2021).

Na pesquisa de Maynard et al., (2021) foi encontrado um dado relevante, cerca de 29,5\% da população estudada foi classificada em sobrepeso, um valor elevado e preocupante, visto que aumenta o risco de desenvolvimento de doenças crônicas não transmissíveis. Por esse motivo, vale ressaltar que a obesidade está diretamente relacionada com uma dieta baseada em alimentos ultraprocessados, como bebidas açucaradas e fast foods, contendo uma alta concentração de substâncias críticas (MARTINS, 2018). Ademais, nas últimas décadas, a população aumentou o consumo desse tipo alimentos e sabe-se que neles contém alta densidade calórica, alta palatabilidade, baixo poder sacietógeno e de fácil absorção e digestão, fatores os quais contribuem e favorecem o aumento da ingestão alimentar, acarretando um desequilíbrio energético (ABESO, 2016). Isto corresponde com o estudo de Aro; Pereira; Bernardo, (2021) no qual foi avaliado a mudança dos hábitos alimentares durante a pandemia, foram notáveis algumas mudanças como um aumento do consumo de alimentos em quantidade exagerada, o comer relacionado com as emoções, consumo de alimentos fora dos horários das refeições e o hábito de "beliscar", com preferência para os doces. Os autores apontam Pesquisa e Extensão 
que todos estes fatores estão relacionados com o aumento de peso da maioria dos participantes durante a pandemia.

Pacientes com sobrepeso ou obesidade podem apresentar sintomas de estresse, como ansiedade, depressão, nervosismo, além de ter o hábito de se alimentar em resposta a problemas emocionais, logo, a partir disso, pode-se sugerir que há relação entre estresse, compulsão por comida palatável, transtorno de compulsão alimentar e obesidade (ABESO, 2016). Isso pode ser observado na pesquisa de Malta et al., (2020) no qual percebeu-se o aumento da ingestão de bebidas alcoólicas em decorrência da ansiedade e tristeza, do medo, insegurança, bem como do risco de morte, aumento no consumo de alimentos não saudáveis durante a semana e ainda houve a redução do consumo dos alimentos saudáveis durante a restrição social acometida pela pandemia da COVID-19 (MALTA et al., 2020). De acordo com Sominsky; Spencer, (2014) o estresse sendo agudo moderado ou crônico moderado pode influenciar o apetite, incluindo impulsos para comer e na escolha do tipo de alimentos.

O estudo de Andrade et al., (2019) mostrou que, no Brasil, há maior consumo de alimentos ultraprocessados quando as refeições são realizadas fora de casa em comparação quando são realizadas apenas em domicílio. Sendo assim, pode-se concluir que com a pandemia e o fechamento de estabelecimentos como restaurantes entre outros pontos comerciais, os cidadãos ao ficarem mais tempo em casa deveriam aproveitar para desenvolver o hábito de realizar preparações de forma mais saudável, contudo, há alguns fatores que poderiam interferir na mudança de comportamentos alimentares menos saudáveis, como a maior dificuldade em obter alimentos frescos, pois demandaria saídas mais frequentes de casa (STEELE et al., 2020).

\section{CONCLUSÃO}

Nesse contexto, os estudos analisados evidenciaram uma relação direta entre impactos na saúde mental e no consumo alimentar durante o período de isolamento social, tendo em vista que pessoas sem alterações na saúde mental, melhoraram a ingestão alimentar, com variedade dos grupos alimentares, menor ingestão de produtos alimentícios industrializados, além do aumento da prática de cozinhar. Entretanto, notou-se também mudanças relacionadas ao ganho de peso, a piora dos hábitos alimentares em pessoas que já apresentavam algum transtorno de ordem mental, com aumento da ansiedade e tristeza.

Os indivíduos diagnosticadas com algum tipo de transtorno de ansiedade, consomem mais alimentos de origem industrializada - seja através de doces, embutidos ou bebidas alcoólicas, a ingestão destes alimentos é uma forma compensatória de lidar com eventos adversos, como o isolamento social, e estados negativos de humor, como o aumento da tristeza e do medo causados pela incerteza da pandemia, além de alterações do estado nutricional que podem agravar a saúde 
mental de indivíduos já afetados.

Desse modo, é necessário mais pesquisas sobre o impacto do isolamento social em decorrência da pandemia da COVID-19, no aspecto mental e alimentar do indivíduo, tendo em vista que os efeitos desencadeados podem permanecer latentes se não houver intervenção adequada e eficaz. Ademais, o estado pandêmico permanece vigente, logo, as consequências geradas por esta situação necessitam de mais estudos para determinar se as conclusões iniciais permanecerão ou serão alteradas por outras consequências associadas à pandemia.

\section{REFERÊNCIAS}

ABESO - ASSOCIAÇÃO BRASILEIRA PARA O ESTUDO DA OBESIDADE E DA SÍNDROME METABÓLICA. Diretrizes brasileiras de obesidade. São Paulo, 2016.

ALONZI, S; TORRE, A. L; SILVERSTEIN, M. W. The Psychological Impact of Preexisting Mental and Physical Health Conditions During the COVID-19 Pandemic. Psychological Trauma: Theory, Research, Practice, and Policy, 2020, Vol. 12, No. S1, S236-S238.

ANDRADE, G; GOMBI-VACA, M; LOUZADA, M; AZEREDO, C; LEVY, R. B. The consumption of ultra-processed foods according to eating out occasions. Public Health Nutrition, v. 23, n. 6, p, 1041-1048, 2020. DOI:10.1017/S1368980019002623.

ARO, F; PEREIRA, B. V; BERNADO, D. N. A. Comportamento alimentar em tempos de pandemia por Covid-19. Brazilian Journal of Development. Curitiba, v.7, n.6, p.59736-59748, 2021.

BANNA, M. H. A. SAYEED, A; KUNDU, S; CHRISTOPHER, E; HASAN, M. T; BEGUM, M. R; KORMOKER, T. DOLA, S. T. I; HASSAN, M. M; CHOWDHURY, S. KHAN, M. S. I. The impact of the COVID-19 pandemic on the mental health of the adult population in Bangladesh: a nationwide cross-sectional study. International Journal of Environmental Health Research.

BARROS, M. B. A. LIMA, M. G; MALTA, D. C; SZWARCWALD, C. L; AZEVEDO, R. C. S; ROMERO, D; JÚNIOR, P. R. B. S; AZEVEDO, L. O; MACHADO, I. E; DAMACENA, G. N; GOMES, C. S; WERNECK, A. O; SILVA, D. R. P; PINA, M. F; GRACIE, R. Relato de tristeza/depressão, nervosismo/ansiedade e problemas de sono na população adulta brasileira durante a pandemia de COVID-19. Epidemiol. Serv. Saúde, Brasília, v. 29, n. 4, 2020.

BRASIL. Boletim epidemiológico especial: doença pelo Coronavírus COVID-19. Semana Epidemiológica 8 (21 a 27/2/2021).

BRASIL. Ministério da Saúde. Secretaria de Vigilância em Saúde. Vigilância de fatores de risco e proteção para doenças crônicas por inquérito telefônico (2006). Brasília, 2007.

BRASIL. Ministério da Saúde. Secretaria de Vigilância em Saúde. Vigilância de fatores de risco e proteção para doenças crônicas por inquérito telefônico (2019). Brasília, 2020.

MAYNARD, D. C; ANJOS, H. A; MAGALHÃES, A. C. V; GRIMES, L. N; COSTA, M. G. O; SANTOS, R. B. Food consumption and anxiety among the adult population during the COVID-19 pandemic in Brazil. Research, Society and Development, v. 9, n. 11, p. e4279119905-e4279119905, 2020. 
SANTOS, R. S. M; AYRES, E. M. Impacto da pandemia de coronavírus (covid-19) no comportamento alimentar. SEMEAR: Revista de Alimentação, Nutrição e Saúde, v. 2, n. 3, p. 2427, 2021.

DURÃES, S. A. SOUZA, T. S; GOMES, Y. A. R; PINHO, L. Implicações da pandemia da covid-19 nos hábitos alimentares. Unimontes Científica, Montes Claro (MG), v. 22, n. 2, p. 1-20, 2020.

LEUCHT, S. A; CIPRIAN, A; FURUKAWA, T. A; PETER, N; PAPAKONSTANTINOU, T; HOLLOWAY, A; SALANTI, G. Living meta-ecological study of the consequences of the COVID-19 pandemic on mental health. European Archives of Psychiatry and Clinical Neuroscience, v. 271, p. 219-221, 2021.

MALTA, D. C; SZWARCWALD, C. L; BARROS, M. B. A; GOMES, C. S; MACHADO, I. E; JÚNIOR, P. R. B. S; ROMERO, D. E; LIMA, M. G; DAMACENA, G. N; PINA, M. F; FREITAS, M. I. F; WERNECK, A. O; SILVA, D. R. P. S; AZEVEDO, L. O; GRACIE, R. The COVID-19 Pandemic and changes in adult Brazilian lifestyles: a cross-sectional study, 2020. Epidemiologia e Serviços de Saúde, v. 29, 2020.

MARTINS, ANA PAULA BORTOLETTO. É preciso tratar a obesidade como um problema de saúde pública. Revista de Administração de Empresas. 2018, v. 58, n. 3, p. 337-341.

OLIVEIRA, L. V; ROLIN, A. C. P; SILVA, G. F; SANTOS, N. R; ARAÚJO, L. C; BRAGA, V. A. L; COURA, A. G. L. Changes in Eating Habits Related to the Covid-19 Pandemic: A Literature Review. Brazilian Journal of Health Review, Curitiba, v.4, n.2, p. 8464-8477, 2021.

POELMAN, M. P; GILLEBAART, M; SCHLINKERT, C; DIJKSTRA, S. C; DERKSEN, E; MENSINK, F; HERMANS, R. C. J; AARDENING, P; RIDDER, D; VET, E. Eating behavior and food purchases during the COVID-19 lockdown: A cross-sectional study among adults in the Netherlands. Appetite, v. 157, 2021.

SCHMIDT, B; CREPALDI, M. A; BOLZE, S. D. A; NEIVA-SILVA, L; DEMENEC, L. M. Saúde mental e intervenções psicológicas diante da pandemia do novo coronavírus (COVID-19). Estud. Psicol., Campinas, v. 37, 2020.

SILVA, R. C. R; PEREIRA, M; CAMPELLO, T; ARAGÃO, E; GUIMARÃES, J. M. M; FERREIRA, A. JF; BARRETO, M. L; SANTOS, S. M. C. Implicações da pandemia COVID-19 para a segurança alimentar e nutricional no Brasil. Ciência e Saúde Coletiva, v. 25, n. 9, p. 3421-3130, 2020.

SMAIRA, F. I; MAZZOLANI, B. C; ESTEVES, G. B; ANDRÉ, H. C. S; AMARANTE, M. C; CASTANHO, D. F; CAMPOS, K. J; BENATTI, F. B; PINTO, A. J; ROSCHEL, H; GUALANO, B; NOCOLETTI, C. F. Poor eating habits and selected determinants of food choice were associated with ultraprocessed food consumption in brazilian woman during the COVID-19 pandemic. Brief research report, v. 8, 2021.

SOMINSKY, E.; SPENCER, S. J. Eating behavior and stress: a pathway to obesity. Frontiers in psychology, vol, 5. n, 434. p, 8. 2014. DOI: 10.3389/fpsyg.2014.00434.

STEELE, E. M.; RAUBER, F.; COSTA, C. dos S.; LEITE, M. A.; GABE, K. T; LOUZADA, M. L. da C.; LEVY, R. B.; MONTEIRO, C. A. Mudanças alimentares na coorte NutriNet Brasil durante a pandemia de covid-19. Revista de Saúde Pública , [S. I.] , v. 54, p. 91, 2020. 